American Journal of Applied Sciences 7 (7): 906-913, 2010

ISSN 1546-9239

(C) 2010 Science Publications

\title{
Effects of Quercetin Encapsulated Liposomes via Nasal Administration: A Novel Cognitive Enhancer
}

\author{
${ }^{1}$ Terdthai Tong-un, ${ }^{1}$ Supaporn Muchimapura, ${ }^{2}$ Wathita Phachonpai and ${ }^{1}$ Jintanaporn Wattanathorn \\ ${ }^{1}$ Department of Physiology, Faculty of Medicine, \\ ${ }^{2}$ Department of Physiology, (Neuroscience Program), Faculty of Medicine, Graduate School, \\ Khon Kaen University, Thailand, 40002
}

\begin{abstract}
Problem statement: Demand for cognitive-enhancing drugs is growing. Numerous medicinal plants possessing antioxidant activity have received much attention as food supplement to improve cognitive function. Quercetin is a potent free radical scavenger and antioxidant. However, the limitations of quercetin: Rapidly metabolized is an obstacle to its use for a cognitive enhancer. In addition, the burden of blood brain barrier can be overcome by nasal administration and liposomes. In the present study, we investigated whether nasal administration of quercetin liposomes could improve spatial memory in healthy adult rats. Approach: Male Wistar rats were pretreated with quercetin liposomes, containing $0.5 \mathrm{mg}$ of quercetin in $20 \mu \mathrm{L}$ (dose $=20 \mu \mathrm{g}$ ), via right nasal cavity once daily continually for 4 weeks. Evaluation of rodent learning and memory was assessed by Morris water maze test and then all rats were sacrificed for determining the survival and cholinergic neurons densities in hippocampus. Results: Quercetin liposomes via nasal route treated rats exhibited a significant improvement in cognitive performance. In addition, nasal administration of quercetin liposomes also resulted in induced the densities of survival and cholinergic neurons in hippocampus. However, further researches about the precise underlying mechanism are still required. Conclusion: Our studies demonstrate that quercetin liposomes via nasal administration may have a candidate for cognitive enhancer in the future.
\end{abstract}

Key words: Quercetin liposomes, nasal administration, antioxidant, blood brain barrier, cognitive enhancer

\section{INTRODUCTION}

Memory is a complex process requiring the coordination of many different regions of brain and many neurotransmitter systems. Among the various neurotransmitter systems, the cholinergic system has attracted special attention because lesions leading to loss of cholinergic neurons in the basal forebrain can cause defects of memory (Leanza et al., 1996; Zhang et al., 1996), muscarinic acetylcholine receptor antagonists when given to animals can induce memory deficit (Introini-Collison and McGaugh, 1988) and agonists (Schwarz et al., 1999) as well as cholinesterase inhibitors (Lyketsos et al., 2004) when given to memory impaired animals or dementia patients can improve the memory.

At present, numerous lines of evidence have demonstrated that many antioxidants can improve cognitive function (Emilien et al., 2000; Kontush and Schekatolina, 2004). During the last few years, antioxidant has received special attention as dietary supplements. Many studies have suggested that reversals in age-related memory declines might be accomplished by increasing the dietary intake possessing high antioxidant activity (Youdim et al., 2002; Andres-Lacueva et al., 2005; Jabeen et al., 2007) and this reversal effect has been claimed to be associated with the antioxidant activity (Raghavendra and Kulkarni, 2001). Thus, a large list of herbal extracts possessing high antioxidant activity and improve memory is now available in the market.

Quercetin $\quad\left(3,5,7,3^{\prime}, 4^{\prime}\right.$-pentahydroxyflavone), a bioflavonoid, frequently found in consumed foods including apples, berries, onions, tea and vegetables (Formica and Regelson, 1995). Indeed, quercetin has many beneficial effects on human health, including cardiovascular protection, anticancer activity, cataract prevention, antiviral activity and anti-inflammatory effects (Nagata et al., 1999). Despite extensive research on the beneficial effects of quercetin in various

Corresponding Author: Terdthai Tong-un, Department of Physiology, Faculty of Medicine, Khon Kaen University, Khon Kaen 40002, Thailand Tel: 66-43-348394 
pathological conditions, the idea of applying quercetin as the cognitive enhancer has become attractive and challenge. In order to apply quercetin for this case, it is necessary to overcome the limitations of quercetin: poor absorption and very low distribution to the brain after oral administration (Boer et al., 2005) due to both rapid metabolism (Manach et al., 1998) and difficulties in the penetration through the Blood Brain Barrier (BBB) (Youdim et al., 2004).

Liposomes have long been used as a Drug Delivery System (DDS) to the brain, because the particles entrap the compounds and prevent rapid degradation elimination or elimination as well as promote penetration through the $\mathrm{BBB}$ and distribution in the brain tissue (Krauze, 2006). Being entrapped in liposomes, a decrease in the dose of a compound to be administered is usually expected (Keller, 2001).

Nasal drug delivery has now been recognized as a very promising route for delivery of therapeutic compounds including biopharmaceuticals. Advantages associated with the nasal administration over oral route include higher bioavailability due to no first pass hepatic metabolism and rapid absorption leading to shorter time to onset of effect (Wang et al., 2006). Here, we investigated whether nasal administration of quercetin liposomes could improve spatial memory in healthy adult rats.

\section{MATERIALS AND METHODS}

High-purity egg L- $\alpha$-phosphatidylcholine, type XVI-E (EPC), cholesterol (chol), quercetin dihydrate (98\% HPLC purity) and Polyethylene glycol 400 (PEG) were purchased from Sigma (Barcelona, Spain). Other reagents used were analytical grade such as chloroform, ethanol and methanol (HPLC and analytical grade) from BDH Laboratory Supplies (Poole, England), disodium hydrogen phosphate, perchloric acid and ortho phosphoric acids (Merck, Darmstadt, Germany), sodium dihydrogen phosphate (JT Baker Inc., Phillipsburg, New Jersey). All other chemicals were at least reagent grade and used as received.

Preparation of quercetin liposomes: Quercetin dehydrate (98\%), high-purity egg L- $\alpha$ phosphatidylcholine, Type XVI-E (EPC) and Cholesterol (CHOL) were prepared as quercetin liposomes. The method used was lipid thin film formation and extrusion (Guo et al., 2003; Liang et al., 2005; Zu et al., 2006).

Animals: Adult male Wistar rats $(180 \pm 20 \mathrm{~g}, 8$ weeks old) were obtained from National Animal Center, Salaya, Nakhon Pathom and they were housed in group of five per cage in standard metal cages at $22 \pm 2^{\circ} \mathrm{C}$ on 12:12 h light-dark cycle. All animals were given access to food and water ad libitum. The experiments were performed to minimize animal suffering in accordance with the internationally accepted principles for laboratory use and care of European Community (EEC directive of 1986; 86/609/EEC).

The experimental protocols were approved by the Institutional Animal Care and Use Committee.

Experimental protocol: All rats were randomly assigned to four groups of eight animals each:

Group 1: Naive intact control

Group 2: Free liposomes: The rats in this group were administered free liposomes via nasal route

Group 3: Liposomes + PEG: The rats in this group were administered PEG which used as vehicle of quercetin liposomes via nasal route

Group 4: Liposomes + Quercetin (QCL): The rats in this group were administered quercetin liposomes via nasal route

All animals were administered freshly prepared quercetin liposomes, containing $0.5 \mathrm{mg}$ of quercetin in $20 \mu \mathrm{L}$ (dose $=20 \mu \mathrm{g}$ ), being drawn into a micropipette, were administered to the right nasal cavity of each rat which was then holding still for $20 \mathrm{sec}$ to ensure the flow and contact of the quercetin liposomes to the nasal cavity whereas the control group received liposomes without quercetin or liposomes + PEG at the same volume once daily for 4 weeks.

Behaviors evaluation: The rats were divided into various groups as mentioned earlier. The behavioral profiles were assessed both after the single dose and repetitive administration of the substance as following: 1-4 weeks of treatment. All animals were submitted to the following behavior tasks (a) Open field test (b) Morris water maze test.

Open field test: In order to assure that cognitive enhancing effect which determined by Morris water maze test was not false positive due to the effect of quercetin liposomes on motor behavior, we also determined the effect of quercetin liposomes on the spontaneous locomotor activity by open field test (Kulkarni and Dandiya, 1973). The open field apparatus was an arena $80 \mathrm{~cm}$ in diameter with a white, opaque wall $30 \mathrm{~cm}$ high. Rats were individually placed in the center of the arena and locomotor activity including the number of grooming, licking and rearing were scored within $5 \mathrm{~min}$. 
Morris water maze test: The water maze consisted of a metal pool $(170 \mathrm{~cm}$ in diameter $\times 58 \mathrm{~cm}$ tall $)$ filled with tap water $\left(25^{\circ} \mathrm{C}, 40 \mathrm{~cm}\right.$ deep) divided into four quadrants. In the center of one quadrant was a removable escape platform below the water level and covered with a nontoxic milk powder. The pool was divided into four quadrants (NE, NW, SE and SW) by two imaginary lines crossing the center of the pool. For each animal, the location of invisible platform was placed at the center of one quadrant and remained there throughout training. The rats must memorize the platform location in relation to various environmental cues because there was nothing directly shows the location of the escape platform in and outside the pool. Therefore, the placement of the water tank and platform were the same in all acquisition trials. Each rat was gently placed in the water facing the wall of the pool from one of the four starting points $(\mathrm{N}, \mathrm{E}, \mathrm{S}$, or $\mathrm{W})$ along the perimeter of the pool and the animal was allowed to swim until it found and climbed onto the platform. During training session, the subject was gently placed on the platform by the experimenter when it could not reach the platform in $60 \mathrm{sec}$. In either case, the subject was left on the platform for $15 \mathrm{sec}$ and removed from the pool. The time for animals to climb on the hidden platform was recorded as escape latency or acquisition time. In addition to the acquisition test, the determination of retention memory was carried out on the next day. According to this test, the platform was removed and the animals were placed into the water maze for $60 \mathrm{sec}$. The retention of memory or the time that the animal spent to swim around the previous location of platform before removing the platform on the test occurring in the next day was also recorded. It has been postulated that if the spatial memory of the rat for the trained platform location is accurate, the rat will swim to the platform location and search around the exact location. Therefore, the more accurate the spatial memory is, the greater the number of times rat swim across the trained platform. In each trial, the animal was quickly dried with towel before being returned to the cage. All test in Morris water maze tests were carried out within $20 \mathrm{~min}$ after the nasal administration of the substances.

Any enhancement of cognition would be reflected by a decrease in escape latency and increase in retention time.

Histological procedure: Following anesthesia with sodium pentobarbital $\left(60 \mathrm{mg} \mathrm{kg}^{-1} \mathrm{BW}\right)$, fixation of the brain was carried out by transcardial perfusion with fixative solution containing $4 \%$ par formaldehyde in $0.1 \mathrm{M}$ phosphate buffer $\mathrm{pH}$ 7.3. The brains were removed after perfusion and stored over a night in a fixative solution that used for perfusion. Then, they were infiltrated with $30 \%$ sucrose solution for approximately $4^{\circ} \mathrm{C}$. The specimens were frozen rapidly and $30 \mu \mathrm{M}$ thick sections were cut on cryostat. They were rinsed in the phosphate buffer and picked up on slides coated with $0.01 \%$ of aqueous solution of a high molecular weight poly L-lysine.

Nissl staining: The duplicate coronal sections of brains were stained with $0.75 \%$ cresyl violet, dehydrated through graded alcohols $(70,95,100 \% 2 \times)$, placed in xylene and cover-slipped using DPX mountant.

\section{Choline acetyltransferase and} immunohistochemistry: A series of section containing hippocampus from each groups were reacted in parallel experiments using a mouse monoclonal antibody detected against Choline Acetyltransferase (ChAT) (Chemicon Internation, Inc., CA, USA) and a modification of a previously described protocol employing the DAKO Strept ABC Complex/HRP duet kit. In brief, the sections were eliminated endogenous peroxidase activity by $0.5 \% \mathrm{H}_{2} \mathrm{O}_{2}$ in methanol. Sections were washed in running tap water and distilled water for $1 \mathrm{~min}$ each, then rinsed in KPBS and KPBS-BT for 5 min per each process. Excess was removed and then incubated for $30 \mathrm{~min}$ in a blocking solution composed of 5\% normal horse serum in KPBS-BT. The sections were then incubated in mouse primary antibody against ChAT diluted 1:100 in KPBS-BT at room temperature for $2 \mathrm{~h}$ and then incubated at $4^{\circ} \mathrm{C}$ for $48 \mathrm{~h}$. The tissue was rinsed in KPBS-BT (two washes $\times 7 \mathrm{~min}$ ), incubated for $4 \mathrm{~h}$ in biotinylated goat antimouse $\mathrm{IgG}$ antibody, rinsed in KPBS-BT (two washes $\times 7 \mathrm{~min}$ ) and then incubated in Strep ABC Complex/HRP for $4 \mathrm{~h}$. In preparation for visualization step, sections were rinsed in KPBS-BT (1 min) and KPBS (two washes $\times 10 \mathrm{~min}$ ). ChAT immunoreactivity was visualized using $0.025 \%$ 3, 3' Diaminobenzidine (DAB, Sigma) and $0.01 \%$ $\mathrm{H}_{2} \mathrm{O}_{2}$. Finally, sections were rinsed in running tap water, air dried and cover-slipped using permount.

Morphological analysis: Five coronal sections of each rat in each group were studied quantitatively. Neuronal counts in hippocampus were performed by eye using a $400 \times$ magnification with final field $255 \mu \mathrm{m}^{2}$ according to the following stereotaxic coordinates: AP-4.8 mm, lateral $\pm 2.4-6 \mathrm{~mm}$, depth 3-8 $\mathrm{mm}$. The observer was blind to the treatment at the time of analysis. Viable stained neurons were identified on the basis of a stained soma with at least two visible processes. Counts were made in five adjacent fields and the mean number extrapolated to give total number of neurons per $255 \mu \mathrm{m}^{2}$. All data are represented as number of neurons per $255 \mu \mathrm{m}^{2}$. 
Statistical analysis: Data are presented as mean \pm Standard Error of Mean ( \pm SEM). One-way Analysis Of Variance (ANOVA), followed by Duncan's post hoc test. A probability level less than 0.05 was accepted as significance.

\section{RESULTS}

Effect of quercetin liposomes on locomotor activity: The effects of quercetin liposomes via nasal administration on spontaneous motor behaviors including grooming, rearing and licking behaviors were demonstrated in Fig. 1-3. The results showed that the spontaneous behavior as mention earlier did not differ significantly between the control, vehicle (PEG), free liposomes treated group and quercetin liposomes throughout the experimental period.

Effect of quercetin liposomes on spatial memory: Hippocampus-dependent spatial memory was assessed with the Morris water maze test. The results showed that vehicle (PEG) and free liposomes treated rats did not differ from their controls either in the escape latency for finding the platform and the retention time. After $20 \mathrm{~min}$ of single administration, quercetin liposomes via nasal administration significantly decreased acquisition and increased retention time $(\mathrm{p}<0.05$ all $)$ as shown in Fig. 4 and 5. In addition, this phenomenon was still observed when the treatment duration was increased to 28 days. This indicates that nasal administration of quercetin liposomes could produce cognitive enhancing effect.

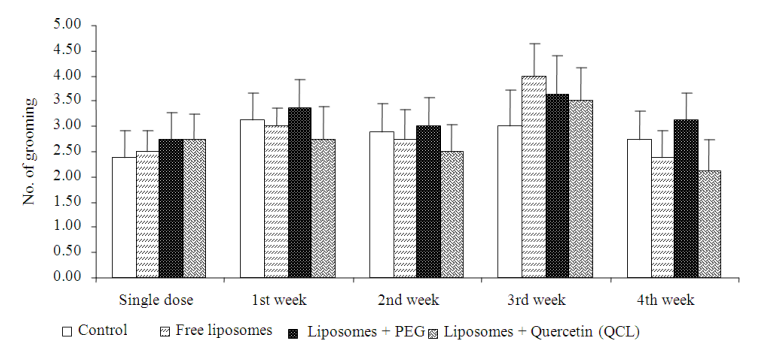

Fig. 1: Effect nasal administration of quercetin liposomes on grooming behaviors $(\mathrm{N}=8)$

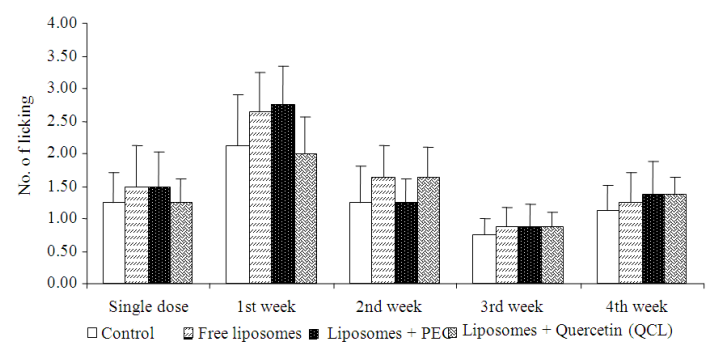

Fig. 2: Effect na + sal administration of quercetin liposomes on licking behaviors $(\mathrm{N}=8)$
Neuroprotective effect of quercetin liposomes via nasal administration: Accumulating data demonstrated that learning and memory particularly spatial memory were tightly associated with the function of various brain areas, which in turn depended on the density of survival neurons particularly cholinergic neurons (Katzman, 1986). Therefore, this study also determined the effect of quercetin liposomes via nasal administration on the alteration of the survival and cholinergic neurons densities in various subregions of hippocampus, the area which played an important role on learning and memory. It was found that the mean \pm SEM survival neurons density in various subregions of hippocampus in vehicle (PEG) and free liposomes treated group were not significantly different from that of control group while the mean \pm SEM survival neurons density in these areas was significantly $(\mathrm{p}<0.05$ all) higher in quercetin liposomes treatment as shown in Fig. 6.

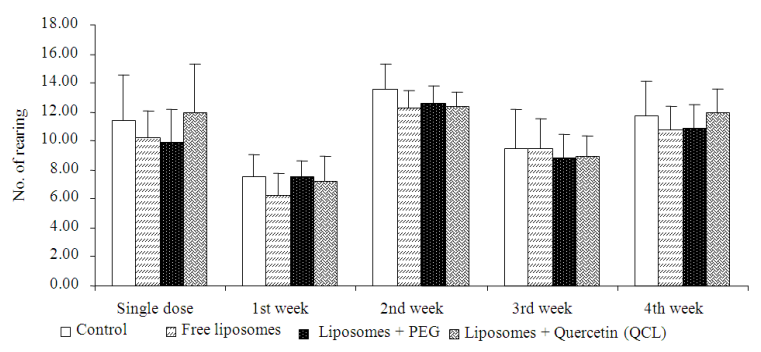

Fig. 3: Effect nasal administration of quercetin liposomes on rearing behaviors $(\mathrm{N}=8)$

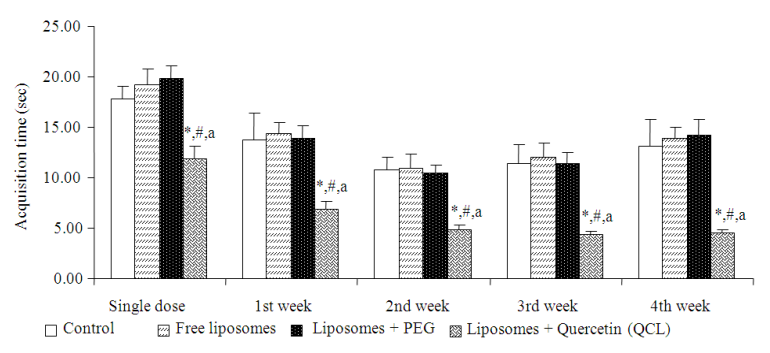

Fig. 4: Effect nasal administration of quercetin liposomes on the acquisition time in the Morris water maze test $(\mathrm{N}=8)$. Results were expressed as mean \pm SEM; ${ }^{*}:$ p-value $<0.05$ compared with control treated group; ${ }^{*}$ : p-value $<0.05$ compared with free liposomes treated group; ${ }^{\mathrm{a}}$ : $\mathrm{p}$ value $<0.05$ compared with liposomes + PEG treated group 


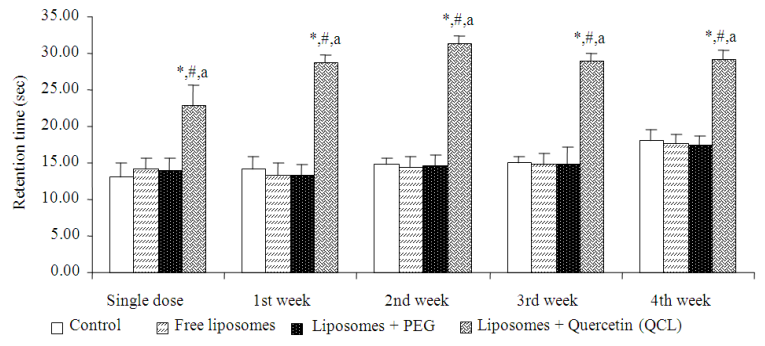

Fig. 5: Effect nasal administration of quercetin liposomes on the retention time in the Morris water maze test $(\mathrm{N}=8)$. Results were expressed as mean \pm SEM. ${ }^{*}:$ pvalue $<0.05$ compared with control treated group; \#: p-value $<0.05$ compared with free liposomes treated group; a: p-value $<0.05$ compared with liposomes + PEG treated group

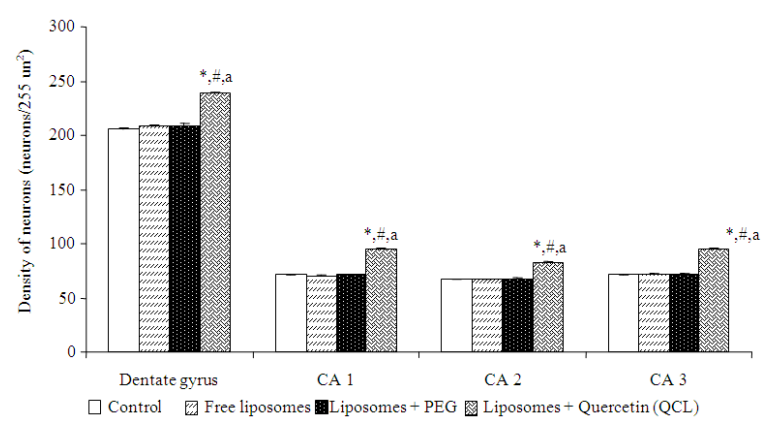

Fig. 6: Effect nasal administration of quercetin liposomes on the survival neurons density in hippocampus. Data were presented as mean \pm SEM. $\quad(\mathrm{N}=8 /$ group $) ;{ }^{*}:$ p-value $<0.05$ compared with control treated group; ${ }^{\#}: \mathrm{p}$ value $<0.05$ compared with free liposomes treated group; ${ }^{a}$ : p-value $<0.05$ compared with liposomes + PEG treated group

In addition, statistical analysis revealed that there were no significant differences in the number of cholinergic neurons density in various subregions of hippocampus in control, free liposomes and vehicle (PEG) treated group while there was a significant induction in cholinergic neurons density of quercetin liposomes treated group in areas as mentioned earlier $(\mathrm{p}<0.05$ all) as shown in Fig. 7 , reflecting the cognitive enhancement via increase in the cholinergic neurons density after nasal administration of quercetin liposomes.

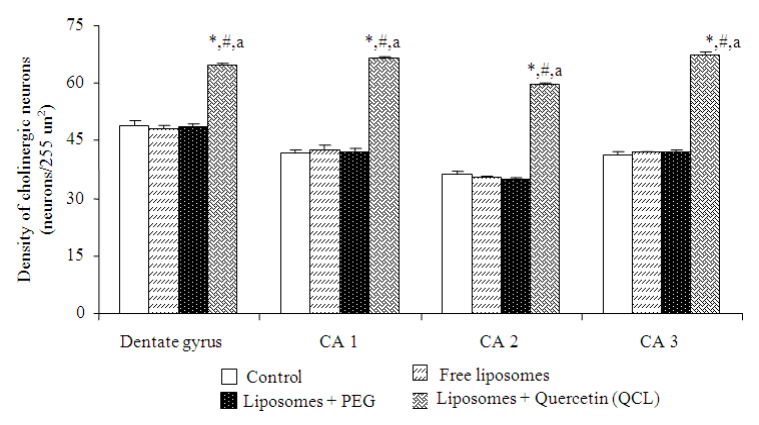

Fig. 7: Effect nasal administration of quercetin liposomes on the cholinergic neurons density in hippocampus. Data were presented as mean \pm SEM. ( $N=8 /$ group). ${ }^{*}$ : p-value $<0.05$ compared with control treated group; \#: p-value $<0.05$ compared with free liposomes treated group; ${ }^{\mathrm{a}}$ : p-value $<0.05$ compared with liposomes + PEG treated group

\section{DISCUSSION}

Here we show that nasal administration of quercetin liposomes could induce the densities of survival and cholinergic neurons in hippocampus resulted in the enhancement of learning and memory in healthy rats.

Recently, a pile of evidence suggested that the prime candidate responsible for producing the neuronal changes mediating the cognitive deficits appeared to be free radicals and oxidative stress generated (Mattson, 2004; Cantuti et al., 2000). Thus, numerous medicinal plants or herbs possessing antioxidant activity have received much attention as food supplement to improve cognitive function. Moreover, treatment with herbal agents is generally cheap and relatively free of side effects (Howes et al., 2003).

Quercetin is a well-known flavonoid distributed ubiquitously in fruits, vegetables and herbs (Havsteen, 1983). Owing to its polyphenolic hydroxyl groups, quercetin exhibits its antioxidant property and is known as a strong free radical scavenger (Rodriguez et al., 2001). Previous studies confirm that quercetin supplementation improve memory deficit condition induced by reserpine in mice (Naumenko and Kulikov, 2006). However, it was reported that quercetin was easily metabolized after absorption (Manach et al., 2004). Clinical studies investigating different programs of administration of quercetin have been limited by its poor water solubility. Many researchers have attempted to improve its solubility by adding Dimethylsulfoxide (DMSO) (Ader et al., 2000). In addition, the safety of the higher DMSO is questionable due to risk of 
vasoconstrictor effect and neurological toxicity (Windrum et al., 2005). It is necessary to develop a strategy, which could provide an elevated pool of antioxidants in the brain region for complete protection of neuronal cells and improve cognitive function. Our study found it worthwhile to use the vesicle mediated carrier system particularly liposomes to deliver flavonoidal antioxidant quercetin to rat brain No unexpected mortality of any animals occurred after nasal administration of quercetin liposomes used in the present study. As a result, vesicles were considered to be safe at the dosing schedule used.

The Morris water maze is commonly used to assess hippocampal-dependent spatial memory in rodents (Morris, 1984). In this task, the rats was treated with quercetin liposomes via nasal route showed significantly decreased acquisition and increased retention time as compared to the control, free liposomes and vehicle (PEG) treated animals, suggested its cognitive enhancing effect.

Moreover, pretreatment with nasal administration of quercetin liposomes significantly increased the density of survival neurons and cholinergic neurons in all areas of hippocampus as compared to the control, free liposomes and vehicle (PEG) treated animals. Therefore, the increased neuron density in hippocampus might involve its neurotrophic action resulted in the neurogenesis.

The neurogenesis could occur throughout adulthood especially in hippocampus and sub ventricular zone of lateral ventricle (Hagg, 2005). Numerous factors had been reported to be regulators of the adult neurogenesis. They also included various neurotransmitters such as dopamine, acetylcholine, serotonin, glutamate and nor epinephrine. All the mentioned neurotransmitters could stimulate the proliferation process (Nacher et al., 2001; Kulkarni et al., 2002; Baker et al., 2005). Recent findings also proposed that the new neuron occurring from the neurogenesis also contributed the important role on learning and memory (Bruel-Jungerman et al., 2007; Kitabatake et al., 2007). However, further study particularly BrdU staining is essential in order to elucidate the neurotrophic effect of quercetin liposomes on neurogenesis in hippocampus resulting in memory improvement.

Our results demonstrated that nasal administration of quercetin encapsulated liposomes was a potential novel cognitive enhancer. It was noticeable that the dose of quercetin required via nasal administration was very much low dose and also produced the rapid onset of cognitive enhancing effect. Based on the previous findings, the decrease effective dose might be associated with many factors including the increase opportunity to transfer directly from the olfactory mucosa along the olfactory pathway to the Central Nervous System (CNS) (Cho et al., 2006) via bypassing $\mathrm{BBB}$, which prevented some CNS-active drugs from reaching the brain (Behl et al., 1998). In addition, it could also decrease the influence of the first pass metabolism (Krauze, 2006). However, the main proposed pathway of quercetin delivery via nasal administration was different. It was likely to permeate through the subarachnoid space through the olfactory epithelium and found in the Cerebro Spinal Fluid (CSF) later, because the liposomes behaved as semilipophilic particles. Therefore, quercetin liposomes could rapidly absorb into the CSF.

\section{CONCLUSION}

Quercetin liposomes via nasal administration to healthy adult rats could increase the densities of survival and cholinergic neurons in the hippocampus. These effects were associated with the enhancement in performance in the Morris water maze behavioral test. Thus, our study, stresses the cognitive enhancement of quercetin liposomes. Despite the fact that the mechanisms underlying these effects are still unknown and require more pharmacological, neurochemical and pharmacokinetic research to establish any therapeutic advantage, quercetin liposomes seem to have the potential to benefit healthy people and contribute to prevention of cognitive decline during aging and neurodegenerative disease.

\section{ACKNOWLEDGEMENT}

This study was partial supported by the National Nanotechnology Center (NANOTEC), NSTDA, National Science and Technology Development Agency, Thailand, through its program of Center of Excellence Network.

\section{REFERENCES}

Ader, P., A. Wessmann and S. Wolffram, 2000. Bioavailability and metabolism of the flavonol quercetin in the pig. Free Radic. Biol. Med., 28: 1056-1067. PMID: 10832067

Andres-Lacueva, C., B. Shukitt-Hale and R.L. Galli et al., 2005. Anthocyanins in aged blueberry-fed rats are found centrally and may enhance memory. Nutr. Neurosci., 8: 111-120. PMID: 16053243

Baker, S.A., K.A. Baker and T. Hagg, 2005. D3 dopamine receptors do not regulate neurogenesis in the subventricular zone of adult mice. Neurobiol. Dis., 18: 523-527. PMID: 15755679 
Behl, C.R., H.K. Pimplaskar, A.P. Sileno, J. de Meireles and V.D. Romeo, 1998. Effects of physicochemical properties and other factors on systemic nasal drug delivery. Adv. Drug Deliv. Rev., 29: 89-116. DOI: 10.1016/S0169409X(97)00063-X

Boer, V.C.J., A.A. Dihal, H. van der Woude, I.C.W. Arts and S. Wolffram et al., 2005. Tissue distribution of quercetin in rats and pigs. J. Nutr., 135: 1617-1618. PMID: 15987855

Bruel-Jungerman, E., C. Rampon and S. Laroche, 2007. Adult hippocampal neurogenesis, synaptic plasticity and memory: Facts and hypotheses. Rev. Neurosci., 18: 93-114. PMID: 17593874

Cantuti, C.I., B. Shukitt-Hale and J.A. Joseph, 2000. Neurobehavioural aspects of antioxidants in aging. J. Neurosci., 18: 367-381. DOI: 10.1016/S07365748(00)00008-3

Cho, J.Y., I.S. Kim, Y.H. Jang, A.R. Kim and S.R. Lee, 2006. Protective effect of quercetin, a natural flavonoid against neuronal damage after transient global cerebral ischemia. Neurosci. Lett., 404: 330-335. PMID: 16806698 DOI: 10.1016/j.neulet.2006.06.010

Emilien, G., K. Beyreuther, C.L. Masters and J.M. Maloteaux, 2000. Prospects for pharmacological intervention in Alzheimer disease. Arch. Neurol., 57: 454-459. PMID: 10768617

Formica, J.V. and W. Regelson, 1995. Review of the biology of quercetin and related bioflavonoids. Food Chem. Toxicol., 33: 1061-1080. PMID: 8847003

Guo, J., Q. Ping, G. Jiang, L. Huang and Y. Tong, 2003. Chitosan-coated liposomes: characterization and interaction with leuprolide. Int. J. Pharm., 260: 167-173. DOI: $10.1016 / \mathrm{S} 0378-$ 5173(03)00254-0

Hagg, T., 2005. Molecular regulation of adult CNS neurogenesis: An integrated view. Trends Neurosci., 28: 589-595. DOI: 10.1016/j.tins.2005.08.009

Havsteen, B., 1983. Flavonoids, a class of natural products of high pharmacological potency. Biochem. Pharmacol., 32: 1141-1148. PMID: 6342623

Howes, M.J., N.S. Perry and P.J. Houghton, 2003. Plants with traditional uses and activities, relevant to the management of Alzheimer's disease and other cognitive disorders. Phytother. Res., 17: 1-18. PMID: 12557240

Introini-Collison, I.B. and J.L. McGaugh, 1988. Modulation of memory by post training epinephrine: Involvement of cholinergic mechanisms. Psychopharmacology, 94: 379-385. PMID: 3128815
Jabeen, B., M. Badaruddin, R. Ali and D.J. Haleem, 2007. Attenuation of restraint induced behavioral deficits and serotonergic responses by stabilized rice bran in rats. Nutr. Neurosci., 10: 11-16. PMID: 17539478

Katzman, R., 1986. Alzheimer's disease. N. Engl. J. Med., 314: 964-973. PMID: 2870433

Keller, B.C., 2001. Liposomes in nutrition. Trends. Food Sci. Technol., 12: 25-31. DOI: 10.1016/S0924-2244(01)00044

Kitabatake, Y., K.A. Sailor, G.L. Ming and H. Song, 2007. Adult neurogenesis and hippocampal memory function: New cells, more plasticity, new memories? Neurosurg. Clin. N. Am., 18: 105-113. PMID: 17244558

Kontush, K. and S. Schekatolina, 2004. Vitamin E in neurodegenerative disorders: Alzheimer's disease. Ann. N. Y. Acad. Sci. 1031: 249-262. PMID: 15753151

Krauze, L., 2006. Real-time imaging and quantification of brain delivery of liposomes. Pharm. Res., 23: 2493 2504. DOI: 10.1007/s11095-0069103-5

Kulkarni, S.K. and P.C. Dandiya, 1973. Effects of antidepressant agents on open field behavior in rats. Psychopharmacologia, 33: 333-338. PMID: 4798172_2

Kulkarni, V.A., S. Jha and V.A. Vaidya, 2002. Depletion of norepinephrine decreases the proliferation, but does not influence the survival and differentiation, of granule cell progenitors in the adult rat hippocampus. Eur. J. Neurosci., 16: 2008-2012. PMID: 12453065

Leanza, G., J. Muir, O.G. Nilsson, R.G. Wiley and S.B. Dunnett et al., 1996. Selective immunolesioning of the basal forebrain cholinergic system disrupts short-term memory in rats. Eur. J. Neurosci., 8: 1535-1544. PMID: 8758961

Liang, M.T., N.M. Davies and I. Toth, 2005. Encapsulation of lipopeptides within liposomes: Effect of number of lipid chains, chain length and method of liposome preparation. Int. J. Pharm., 301: 247-254. PMID: 16054787 DOI: 10.1016/j.ijpharm.2005.06.010

Lyketsos, C.G., W.E. Reichman, P. Kershaw and Y. Zhu, 2004. Long term outcomes of galantamine treatment in patients with Alzheimer disease. Am. J. Geriatr. Psychiat., 12: 473-482. PMID: 15353385

Manach, C., C. Morand, V. Crespy, C. Demignee, O. Texier and F. Reegeerat et al., 1998. Quercetin is recovered in human plasma as conjugated derivatives which retain antioxidant properties. FEBS Lett., 426: 331-336. PMID: 9600261 
Manach, C., A. Scalbert, C. Morand, C. Remesy and L. Jimenez, 2004. Polyphenols: Food sources and bioavailability. Am. J. Clin. Nutr., 79: 727-747. PMID: 15113710

Mattson, M.P., 2004. Pathways towards and away from Alzheimer's disease. Nature, 430: 631-639. PMID: 15295589

Morris, R.G.M., 1984. Developments of a water-maze procedure for studying spatial learning in the rat. $\mathrm{J}$. Neurosci. Methods, 11: 47-60. PMID: 6471907

Nacher, J., D.R. Rosell, G. Alonso-Llosa and B.S. McEwen, 2001. NMDA receptor antagonist treatment induces a long-lasting increase in the number of proliferating cells, PSA-NCAMimmunoreactive granule neurons and radial glia in the adult rat dentate gyrus. Eur. J. Neurosci., 13: 512-520. PMID: 11168558

Nagata, H., S. Takekoshi, T. Honna and K. Watanabe, 1999. Antioxidative action of flavonoids quercetin and catachin mediated by the activation of glutathione peroxidase. J. Exp. Clin. Med., 24: 1-11. PMID: 10530620

Naumenko, V.S. and A.V. Kulikov, 2006. Quantitative assay of 5-HT (1A) serotonin receptor gene expression in the brain. Molekuliarnaia Biol., 40: 37-44. PMID: 16523690

Raghavendra, V. and S.K. Kulkarni, 2001. Possible antioxidant mechanism in melatonin reversal of aging and chronic ethanol-induced amnesia in plusmaze and passive avoidance memory tasks. Free Radic. Biol. Med., 30: 595-602. PMID: 11295357

Rodriguez, R.J., C.L. Miranda, J.F. Stevens, M.L. Deinzer and D.R. Buhler, 2001. Influence of prenylated and non-prenylated flavonoids on liver microsomal lipid peroxidation and oxidative injury in rat hepatocytes. Food Chem. Toxicol. 39: 437-445. PMID: 11313109
Schwarz, R.D., M.J. Callahan and L.L. Coughenour et al., 1999. Milameline (CI979/RUI35926): A muscarinic receptor agonist with cognition activating properties: Biochemical and in vivo characterization, J. Pharmacol. Exp. Ther. 291: 812-822. PMID: 10525104

Wang, X., H. He, W. Leng and X. Tang, 2006. Evaluation of brain-targeting for the nasal delivery of estradiol by the microdialysis method. Int. J. Pharm., 317: 40-46. PMID: 16631329

Windrum, P., T.C. Morris, M.B. Drake, D. Niederwieser and T. Ruutu, 2005. Variation in dimethyl sulfoxide use in stem cell transplantation: A survey of EBMT centers. Bone Marrow Transplant., 36: 601-603. PMID: 16044141

Youdim, K.A., J.P. Spencer, H. Schroeter and C. Rice-Evans, 2002. Dietary flavonoids as potential neuroprotectants. Biol. Chem., 383: 503-519. PMID: 12033439

Youdim, K.A., M.Z. Qaiser, D.J. Begley, C.A. Rice-Evans and N.J. Abbott, 2004. Flavonoid permeability across an in situ model of the bloodbrain barrier. Free Radic. Biol. Med., 36: 592-604. PMID: 14980703

Zhang, Z.J., T.G. Berbos, C.C. Wrenn and R.G. Wiley, 1996. Loss of nucleus basalis magnocellularis, but not septal, cholinergic neurons correlates with passive avoidance impairment in rats treated with 192-sporin, Neurosci. Lett., 203: 214-218. PMID: 8742031A

Zu, Y., C. Li, Y. Fu and C. Zhao, 2006. Simultaneous determination of catechin, rutin, quercetin kaempferol and isorhamnetin in the extract of sea buckthorn (Hippophae rhamnoides L.) leaves by RP-HPLC with DAD. J. Pharm. Biomed. Anal., 41: 714-719. PMID: 16520013 DOI: 10.1016/j.jpba.2005.04.052 